\title{
Determining levels of biological geometric information at polygonal shape patterns.
}

\author{
Philipp von Bülow 2, Jose Gerardo Carrillo Gonzalez 3, Carlos Ortega Laurel 3, Gerardo Laguna Sanchez 3, Jacobo \\ Sandoval Gutierrez ${ }^{2}$, Francisco Perez-Martinez ${ }^{3}$, Kalina Miranda-Perkins ${ }^{4}$ and Juan Lopez-Sauceda ${ }^{1,2, *}$. \\ 1 Mexican National Council for Science and Technology (CONACYT, Mexico). Full postal address; Av. \\ Insurgentes Sur 1582, Col. Crédito Constructor, Zip code 03940, Mexico City, Mexico ; e-mail@e-mail.com \\ 2 Department of Productive Processes, Metropolitan Autonomous University. Full postal address; Av. \\ Hidalgo Pte. 46, Col. La Estación, Zip code 52006, Lerma de Villada, Estado de Mexico, Mexico. \\ 3 Department of Information Systems and Communication, Metropolitan Autonomous University. Full postal \\ address; Av. Hidalgo Pte. 46, Col. La Estación, Zip code 52006, Lerma de Villada, Estado de Mexico, Mexico. \\ 4 Postgraduate College (COLPOS). Full postal address; Km. 36.5, Road to Texcoco, Zip code 56230, \\ Montecillo, Estado de Mexico, Mexico. \\ * Correspondence: Juan López-Sauceda (j.lopez@correo.ler.uam.mx); +052 (Country code) 5553760012.
}

\begin{abstract}
Based on a measuring system to determine the statistical heterogeneity of individual polygons we propose a method to use polygonal shape patterns as a source of data in order to determine the Shannon entropy of biological organizations. In this research, the term entropy is a particular amount of data related with levels of spatial heterogeneity in a series of different geometrical meshes and sets of random polygons. We propose that this notion of entropy is important to measure levels of information in units of bits, measuring quantities of heterogeneity in geometrical systems. In fact, one important result is that binarization of heterogeneity frequencies yields a supported metric to determine geometrical information from complex configurations. Thirty-five geometric aggregates are tested; biological and non-biological, in order to obtain experimental results of their spatial heterogeneity which is verified with the Shannon entropy parameter defining low particular levels of geometrical information in biological samples. Geometrical aggregates (meshes) include a spectrum of organizations ranging from cell meshes to ecological patterns. Experimental results show that a particular range (0.08 and 0.27$)$ of information is intrinsically associated with low rates of heterogeneity. We conclude it as an intrinsic feature of geometrical organizations in multi-scaling biological systems.
\end{abstract}

Keywords: Biocomplexity 1; Geometrical heterogeneity 2; Information theory 3.

1. Introduction In the context of shapes and forms in biology there has been an historical effort to find the source of some biological patterns and the fundamental nature of their seemingly steady element arrangement. Traditionally, the bottom-up logic of biological developing structures as a dynamical time-space expression process has been extensively approached either by neodarwinism (i. e. genetic blueprint or functional viewpoints) or by biological structuralism (e. g. fractal biologic patterns from chaos theory). In fact, these sort of approaches are supported by epistemological conceptions defining traditions of research work, such as that positioning the whole organisms made of atomic and separate parts and their history (i.e., systematics) or the holistic dynamical system approach of the structuralism point of view (i.e., Turing patterns). In contrast, our perspective includes the use of Shannon entropy to understand biological organizations as a geometric whole whose configurations defining their steady state result from an inherent and specific associated level of information. One important example of that steady state is derived from a prevailing and well stereotyped distribution of cellular polygons conserved in proliferating 
metazoans tissues (i. e. epithelium). Whether patterns and shapes are an active source of geometrical information, stability and variability during developmental process and evolution or not is a topic that from our angle has been neglected. On the other hand, some distant perspectives have been throughout the path of deep questions about shapes [1] beside some outstanding logics of architectural order in nature [2]. In fact, all of those questions had endorsing important inferences for academic areas such as, architecture [2], cell geometry [3], [4], material sciences [5], medicine [6-9], even aesthetics [10], philosophy [2] and arts [11]. In addition, the mathematical perspective of topology, dynamical systems and other important areas related with geometry, statistics and discrete mathematics have been involved in a lot of important achievements [12-15], mainly tracked by new emerging questions rather than achievements of solid answers. In some sense, almost all those researches are in the race of searching for particular models of construction for biological forms. Nevertheless, in the context of this paper, the geometric properties of individual discrete elements in forms are not simple intrinsic features biologically exposed as outcomes. Instead, we understood them as both, independent spaces into a larger whole and as unities defining interacting properties inside of a biocomplex larger whole of geometric information. In this line, our main methodological question about shapes arises: There is a way to quantify geometrical order and information in biological organizations? In some sense, it is not a novel question. There is an important amount of works related with that issue of quantifying information at different complexity levels in biological networks [16-19], ecosystems [20-24], molecular entropy [25] and cellular entropy [26], just to mention some. Also, forms of spatial entropy looking for the characterization of ecological landscape heterogeneity, such as urban, sociological and economical properties at multiple scales associated with them have been broadly approached [27-29], using some geometrical tools. However, in the context of pure biology the underlying informational order behind the geometry of general biological organizations is still lacking. There are some intuitions regarding quantitative values matching biology and architecture through some of the references [2], [30]. Nevertheless, the limits defining quantitative parameters of order according to entropy, as a generic value for biological organizations, is still an issue able to be solved. We consider that one important obstacle in that determined research direction is the establishment of a set of biological forms of reference to start to work on.

The establishment of the core idea to measure spatial heterogeneity to determine the geometrical entropy of a particular biological form is derived from a previous analysis of geometrical constrictions in five-fold morphologies (polygonal random disc organizations [30]). In that work, we found that spatial organization of five-fold morphologies is statistically lower than all other planar disc organizations studied there (three to ten-fold morphologies) in terms of spatial heterogeneity (unequal distribution of space inside polygons). In fact, we found a statistical basin corresponding to the most frequent morphologies in biological disc organizations (three, four, five and six disc partitions [30]). On the other hand, whereas we found a statistical value to approach the key idea of low heterogeneity for related morphologies in nature, we did not have a parameter to work on to reach a method to capture quantitatively the geometrical limits of biological organizations with a formal framework of reference. It is important to say that a lot of work has been done regarding the larger geometrical context of cells and the physical causalities of interactions into cell aggregates [31-37], which notably enforces our background. However, all of this work derives from physical parameters, describing some geometrical properties and not strictly determining levels of geometric information. In addition, the characterization of self-assembled 2D Patterns with Voronoi Entropy in [38-39], also represents some sort of approaches about levels of generic heterogeneity at different scales achieving geometry as a source of organization. In 
spite of that, the main results of that kind of works release some ideas about entropy in a context of matter organization and ecological dynamics motivating some researches on material sciences and territorial aspects, without go deep into questionings about the nature or the informational limits of generic order in biological shapes.

The outline of an area or figure is a shape that can be a determined configuration of discrete elements, which sometimes can be understood as a population of geometric parts as constitutive elements. Our approach here is the establishment of a method to determine levels of geometric information at polygonal shape patterns (PSP) using Shannon entropy as the main theoretical framework. Informational entropy allows the quantification of order and disorder levels from discrete and continuous geometric variables. Accordingly, the first focus of our view regards on extract basic discrete and continuous geometric principles of polygons immersed into larger whole organizations (PSP), in order to standardize levels of biological information given several amounts of heterogeneity. Rather than just on looking at polygons and their aggregates as mathematical outcomes derived from computing simulations we developed an statistical process to detect levels of information from pure geometry. Our first results find that spatial heterogeneity of polygons (unequal distribution of space inside polygons) represents a free scale approach that can be generalized on a wide range of biological size scales, which also, can be easily translated into an informational entropy metric model. Hence, the second part of our approach will be the use of the Shannon entropy parameter associated with frequencies of that spatial heterogeneity of polygons to define biological limits in terms of information. Finally, we determine a measure system for a significant spectrum of geometric living and non-living configurations. Generally, the statistical recurrence of geometric patterns in certain complex architectures, seen from its resulting distribution [40], codifies emergent key properties such as information and self-organization [28]. On this research work, we seek to establish a novel connection, between information theory [41] and the architecture of biological organizations in order to capture biological systems information keeping a formal parameter of reference to retrieve geometric information from generic forms. In biological terms, there is a not logical association the relation between self-organization and the reduction of amounts of information in a system. Intuitively, biological configurations should have a lot of information in contrast with non-biological configurations. In fact, highly ordered organizations such as hexagonal structures store less Shannon entropy due to the high level of ordering in their cluster arrangements [38]. In contrast, several entropy metrics of random samples determining levels of spatial heterogeneity in the context of landscape patterns indicates incremental values of information [39]. Living systems shows an important reduction of entropy reaching very low values beside selforganization as an ostensibly consistent rule [5], [6], [9], [16], [18], [21], [25], [28], [30], [38], [39]. Such behavior has been associated with biological and physical constraints [42-43] with some intuitions regarding pure geometry [1], [4], [5], [6], [9], [40], [44], which is the focus that we will try to verify on this work. The more self-organized is a system, the less entropic its behavior. Hence, we expect that information will decrease along the ordering of geometric parts in potentially dissipative structures.

Our material of work will be areas (polygons) and their associated sub-areas (triangles) defining levels of heterogeneity and their frequency. Our procedure satisfies the fact that we may work with polygonal shapes as a method that can discern between levels of geometrical heterogeneity using a width spectrum of statistical variation. The Shannon entropy is a parameter indicating information that is a clear resolution of uncertainty. This metric will be tested into meshes (biological, nonbiological and random polygonal arrangements) extracted from the web and random polygons with different number of sides. Finally, we will bring back the informational 
limits of biological structures whose geometry, potentially, would be biologically representative in terms of their closeness with nature images due to the informational entropy associated.

To develop the idea, this paper is organized as follows: There is a first exposition of the collecting method, features and categorization of biological images samples and non-biological mesh simulations in section (2). All of this data will define the material to work on besides random polygons with different number of sides. The mathematical model and the statistical motivation to work on these polygons and the main background to define heterogeneity in spatial organization of polygonal shapes and meshes are given in Section (2.2) and (2.3) of methods. The binarization, the most practical and explicit procedure to measure the quantity of information in geometrical meshes that we have found in biological and non-biological systems using Shannon entropy, as well as the associated statistical distributions of internal partitioning in shapes $\boldsymbol{\Gamma}$, is determined as a result in section (3). Finally, section 4 and 5 corresponds to discussion and conclusions, respectively.

\section{Materials and Methods}

Our first step was the establishment of a collection of nature images and sample data to work on. Several biological cell organizations have been used as models to define geometric parameters. In that sense, an important number of studies have analyzed the topological properties of many cell organizations [3], [4], [5], [6], [9], [32], [33], [34], [35], [36], [40], [44], [45], [46], [47], [48]. Also, a lot of epithelium models have extensively used anatomical parts, developmental stages and tissue variations images. In fact, there is a prevailing and well stereotyped distribution of cellular polygons (SDCP) conserved in proliferating metazoans tissues with a polygonal frequency of $29 \%$ of 5 -sided polygons, $49 \%$ of 6 -sided polygons, and $20 \%$ of 7 -sided polygons [34], [40], [45]. In that context, some other images of biological cell organizations are able on line, such as, histological samples derived from different human tissues [34], [46]. Currently, it is widely accepted that although variation in those organizations exist there is just a narrow range of variations of cellular polygonal distributions [34], [40]. In this regard, samples of polygonal mesh are directly comparable even if these meshes are from different nature or scales [49], [50] due to all of them are PSP, including biological natural images, biological simulations, non-biological simulations, such as random meshes and Poisson-Voronoi tessellations and random polygons. Therefore, levels of Shannon entropy in polygonal meshes and set of random polygons turns out into a window of universal and comparable information if we approach them from a pure geometric perspective.

\subsection{Collecting samples on line}

We collect samples of images on line looking for a broad and representative set of biological organizations in order to support our main hypothesis: Geometric information defined by the Shannon entropy of spatial polygonal heterogeneity is a proper parameter able to define the limits of a generic biological organizational value using PSP. Thus, the establishment of a measure of spatial organization able to determine the geometrical entropy of order for biological forms must be analyzed measuring biological and non-biological organizations (Figure 1). At tissue level, we used images from proliferating Drosophila prepupal wing discs (dWP); [34], [45], [47], middle third instar wing discs (dWL); [45], [47], normal human biceps (BCA); [34], muscular dystrophy from skeletal muscles (MD); [46] and pseudo stratified Drosophila wing disk epithelium (PSD); [40]. Also, at the ecological level polygonal meshes derived from Namibia fairy circles (ecological patterns associated with SDCP convergences [49]) images were integrated into the analysis (NFC); [49], [50], [51]. The 
global tag to define MD, dWP, dWL, BCA, PSD and NFC is called BIO. The nonbiological meshes were different diagrams resulting from different vertex model simulations. All of those simulations were based on quantified distances from SDCP which is traditionally used as reference in epithelial studies [52], [53], [54]. According to reference [34], the closeness with SDCP can be defined using optimal paths approaching by using iterations of Lloyd's algorithm and investigating the effects of cell divisions on topology. In contrast, reference [44] reached equilibrium states by seeking minimal potential energy. Given this, there were epithelium simulations which we defined as control simulations (CS); [34], [43], simulation out of equilibrium (SOE); [34], [43], simulation at equilibrium (SAE); [34], [43], atrophy simulation (AS); [34] and Poisson-Voronoi tesselation (PT); [34]. We consider CS, SOE, SAE, AS and PT altogether as non-biological meshes (Non BIO), since they were derived from algorithms. Finally, we include an algorithmic routine from reference [49] to develop random arrangements [RA] into the global analysis as a control. Therefore, we have three mesh categories, BIO, Non BIO and RA.
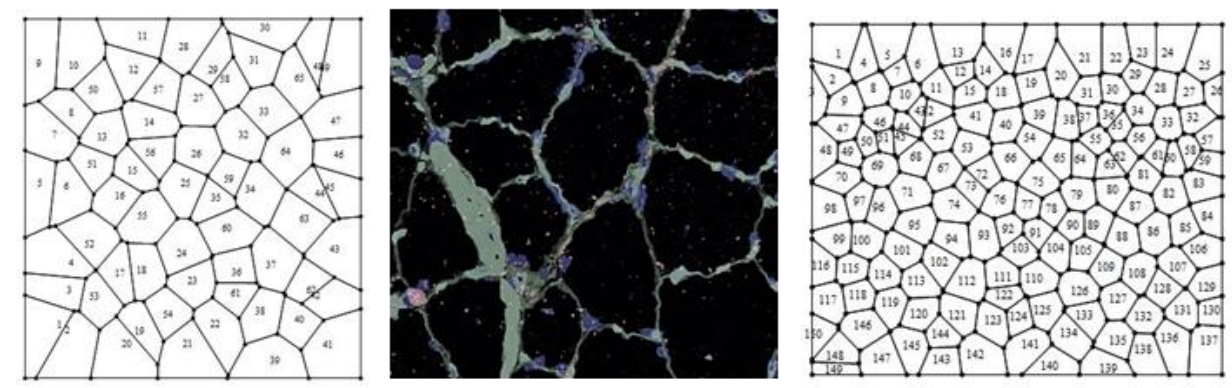

Figure 1. Three general types of mosaics were analyze. Left side; random arrangements (RA), center; Center; natural images (BIO, either stained or schematic aggregates of cells and ecological polygonal meshes) extracted from the web; muscular dystrophy (MD), Drosophila prepupal wing discs (dWP), middle third instar wing discs (dWL), normal human biceps (BCA), pseudo stratified Drosophila wing disk epithelium (PSD) and ecological patterns (NFC; main text). Right; processed non biological images (Non BIO) extracted from the web which we named, control simulation (CS), simulation at equilibrium (SAE), atrophy simulation (AS), simulation out of equilibrium (SOE), and Poisson-Voronoi tessellation (PT).

\subsection{Heterogeneity of spatial organization in shapes $\Gamma$.}

The establishment of a measure of spatial organization able to determine the geometrical entropy of biological organizations is derived from a previous analysis of geometrical constrictions in five-fold morphologies [30]. In this new work our main methodology goes beyond centered on statistical measurements of geometrical heterogeneity onto biological PSP, associating particular levels of entropy to them. Therefore, the main aim of our methodology regards for determining levels of heterogeneity in biological PSP and their associated information.

A former statistical analysis is derived from the study of partitions (areas) and their sub-localities (sub-areas) arising from constructions generically named shapes $\boldsymbol{\Gamma}$. A shape $\boldsymbol{\Gamma}$ is a set of spatial planar confined regions (group of polygons) called sublocalities inside a partition $\boldsymbol{P}_{\boldsymbol{i}}$ (each individual polygon). Therefore, a shape $\boldsymbol{\Gamma}$ could be a set of regular or irregular polygon with a determined number of sides. On that sense, each shape $\boldsymbol{\Gamma}$ is a set of areas that can be subject to be statistically analyzed. The main idea to establish the generic name of shape $\boldsymbol{\Gamma}$ is due to it is useful to name both polygons (e. g. irregular hexagon and regular ones) and/or areas (numeric values) associated with particular shapes. 
Each locality $\boldsymbol{P}_{\boldsymbol{i}}$ is constituted by a subset of a given number $\boldsymbol{N}_{\boldsymbol{i}}$ of sub-localities, $\boldsymbol{S}_{\boldsymbol{i 1}}, \boldsymbol{S}_{\boldsymbol{i} 2}, \ldots, \boldsymbol{S}_{\boldsymbol{i}_{\boldsymbol{N} i}}$ such that $\boldsymbol{P}_{\boldsymbol{i}}=\mathrm{U}_{\boldsymbol{j}=\mathbf{1}}^{\boldsymbol{N}_{\boldsymbol{i}}} \boldsymbol{S}_{\boldsymbol{i j}}$, where $\boldsymbol{P}_{\boldsymbol{i}}$ is a spatial region which could be a convex polygon in $\mathbb{R}^{2}$. Let $\boldsymbol{A}_{\boldsymbol{i} \boldsymbol{j}}$ be the area of each sub-locality. If $\boldsymbol{A}_{\boldsymbol{i} \boldsymbol{j}}=\boldsymbol{A}_{\boldsymbol{i} \boldsymbol{k}} \forall \boldsymbol{k}, \boldsymbol{j}$, then we said that $\boldsymbol{P}_{\boldsymbol{i}}$ is non heterogeneous (Figure 2). In contrast, if exists some $\boldsymbol{j} \neq \boldsymbol{k}$ such that $\boldsymbol{A}_{\boldsymbol{i j}} \neq \boldsymbol{A}_{\boldsymbol{i} \boldsymbol{k}}$ then we say that $\boldsymbol{P}_{\boldsymbol{i}}$ is heterogeneous. Therefore, let $\boldsymbol{A}_{\boldsymbol{i}}=\sum_{\boldsymbol{j}=\mathbf{1}}^{\boldsymbol{N}_{\boldsymbol{i}}} \boldsymbol{A}_{\boldsymbol{i} \boldsymbol{j}}$ be the sum of all the associated areas of every partition; this set determines a polygon or shape $\boldsymbol{\Gamma}=$ $\left\{\boldsymbol{A}_{\boldsymbol{i}}\right\}$. Therefore, $\boldsymbol{\Gamma}$ is a generalization of an area with any set of sub-areas which are considered in numerical terms.

Therefore, the area average of a partition $\boldsymbol{P}_{\boldsymbol{i}}$ is:

$$
\bar{A}_{i}=\frac{1}{N_{i}} \sum_{j=1}^{N_{i}} A_{i j}
$$

and

$$
\sigma_{i}=\sqrt{\frac{1}{N_{i}-1} \sum_{j=1}^{N_{i}}\left(A_{i j}-\overline{A_{\imath}}\right)^{2}}
$$

is the standard deviation of each partition. Notice that if $\boldsymbol{\sigma}_{\boldsymbol{i}}=\mathbf{0} \Rightarrow \boldsymbol{A}_{\boldsymbol{i j}}=\boldsymbol{A}_{\boldsymbol{i} \boldsymbol{k}} \forall \boldsymbol{j}, \boldsymbol{k}$; therefore that partition is non heterogeneous.

The next equation reflects the amount of heterogeneity in a given shape $\boldsymbol{\Gamma}$,

$$
x_{i}=\sigma_{i} / \bar{A}_{i}
$$

we include $\overline{\boldsymbol{A}}_{\boldsymbol{i}}$ in order to normalize the data and constraint the heterogeneity outcomes inside a scale from 0 to 1 .

a)

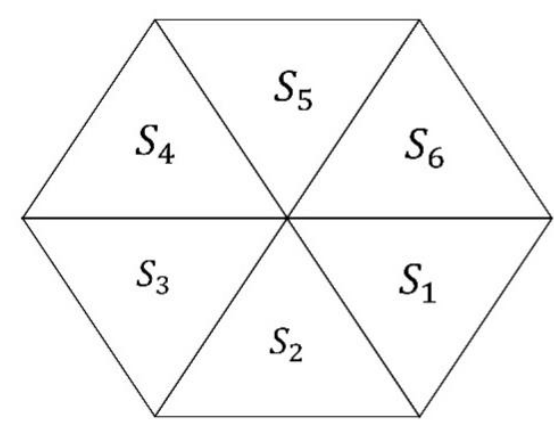

b)

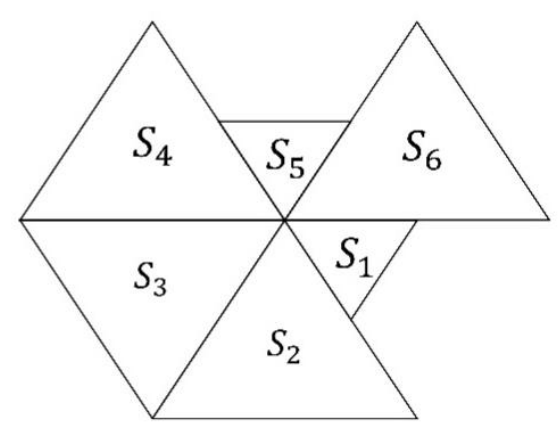


Figure 2. Schematic properties of two different shapes $\boldsymbol{\Gamma}$. a) A regular hexagon is a partition associated with six sub-areas from six sub-localities $\boldsymbol{S}_{\mathbf{1}}, \boldsymbol{S}_{\mathbf{2}}, \ldots, \boldsymbol{S}_{\mathbf{6}}$ which are all equal then is non heterogeneous. b) A shape $\boldsymbol{\Gamma}$ with a six-fold partition such that any of their sub-localities have unequal subareas is heterogeneous; the set of areas defined by sub-localities $\boldsymbol{S}_{\mathbf{1}}$ and $\boldsymbol{S}_{\mathbf{5}}$ are smaller than those of $\boldsymbol{S}_{2}, \boldsymbol{S}_{\mathbf{3}}, \boldsymbol{S}_{\mathbf{4}}$ and $\boldsymbol{S}_{\mathbf{6}}$.

The main objective of our research here is the establishment of a measure of spatial organization able to determine the geometrical entropy of biological and nonbiological organizations. Therefore, we relate equation (3) with a proper collection of data reflecting rates of spatial heterogeneity, quantifying indexes of heterogeneity in all of our theoretical samples. Consequently, our results will be the standard deviation of heterogeneity derived from levels of variability in a collection of PSP samples (section 2.1) and shapes $\boldsymbol{\Gamma}$ (section 2.2). In order to define the standard deviation of heterogeneity we have to determine first the average of PSP heterogeneity (equation 4) for all samples,

$$
\bar{x}_{i}=\frac{1}{N_{s}} \sum_{j=1}^{N_{s}} x_{i s}
$$

where the second subscript corresponds to the number of shape $\boldsymbol{\Gamma}$ sample. Given this equation the standard deviation can be obtained,

$$
\sigma_{x i}=\sqrt{\frac{1}{N_{s}-1} \sum_{j=1}^{N_{s}}\left(x_{i s}-\bar{x}_{i}\right)^{2}}
$$

According to this last equation the standard variation of heterogeneity will reflect a global statistical value to determine area variability and define the informational entropy using geometry.

\subsection{Heterogeneity of polygonally shape patterns.}

An important fact before defining entropy in mosaics of cells is related with the developing of a proper methodology to get the coordinates of individual polygons. In fact, as we mention in section 2.1 we used natural (biological) and non-biological processed images extracted from different web sites and references to define the coordinates of polygons using the centroid of each polygon as the origin of polygonal coordinates. Spatial heterogeneity in mosaics of polygons was derived using equation ( $1 \& 2)$ to obtain the heterogeneity for each polygon using equation (3; Figure 3). With this data is possible to get a statistical frequency distribution of heterogeneities for each mesh. 


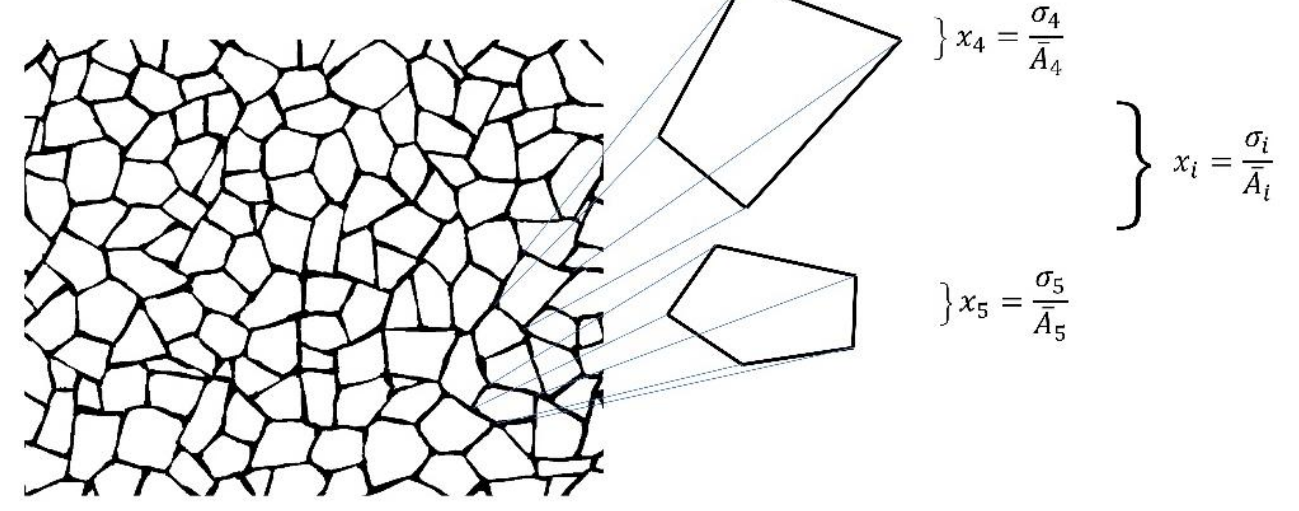

Figure 3. Symbology of equations for individual polygons extracted from a mesh. The generic expression given by equation 3 is used in order to obtain individual polygon heterogeneity. Also, the distribution of spatial heterogeneity derived from frequencies levels of heterogeneity in meshes of polygons of BIO, Non BIO and RA was defined using the values given by heterogeneity equation. Generalization for equations defining heterogeneity of polygons is written $\boldsymbol{x}_{\boldsymbol{i}}$, where sub-index $\boldsymbol{i}$ reflects the number of sides.

\section{Results}

\subsection{Continuous distribution of heterogeneity.}

A parallel but important arising question is whether the variable side number of polygonal shapes in the context of heterogeneity might lead to a continual progression in terms of informational amount or not. That is entropy as a function of polygonal side number. Since meshes are sets of mixed polygons with different number of sides this question must be approached using frequency distributions of heterogeneity in shapes $\Gamma$ using polygons derived from number of sides as the independent variable. Initially, we have to transform non-continuous distributions of frequencies into continuous ones using heterogeneity data applying a probability density function (Wolfram Mathematica 9.0; Figure 4). 


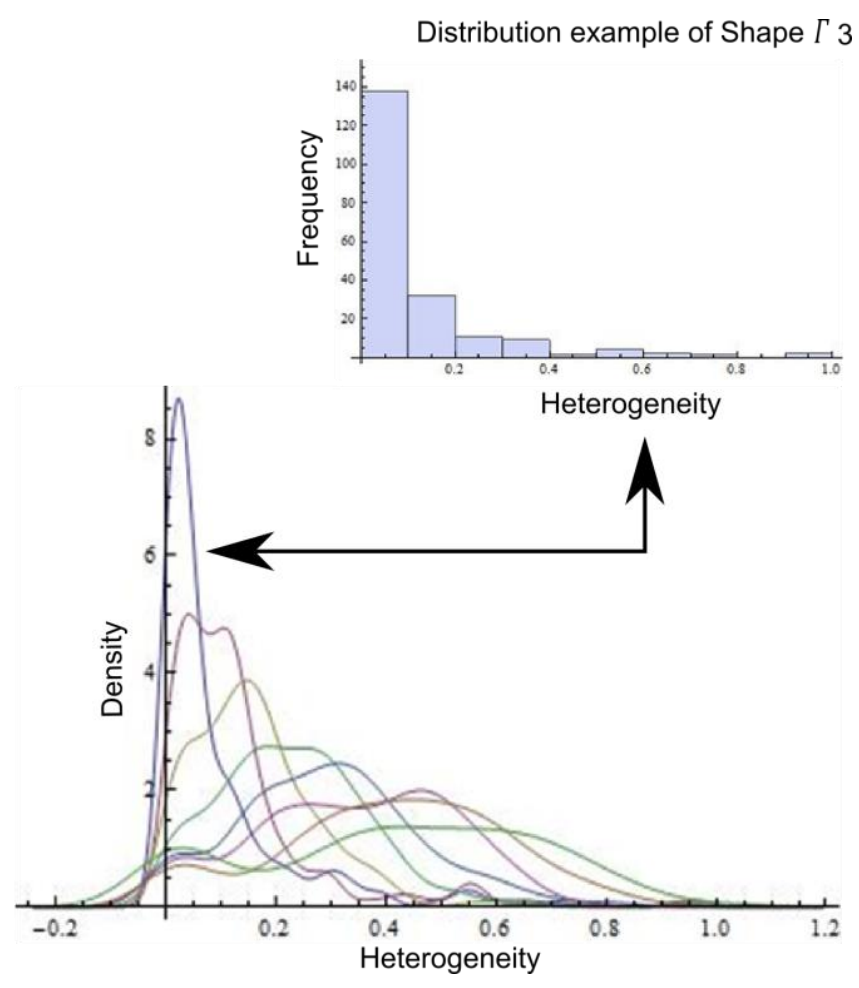

Figure 4. Frequency distributions of random polygons. Upper figure shows an example of frequency values for Shapes $\Gamma$ derived from random polygons with three sides. Bottom figure shows probability density function applied to transform histograms derived from all random polygons (3-10) with discrete values into continuous graphics.

In order to start with a continuous approach to detect levels of entropy we decided to use those distributions to exert a Kolmogorov-Smirnov test (KST) to detect distribution differences between normal distributions and the remaining ones. According to figure 5, the Log base 10 values of a Kolmogorov Smirnov test applied in Wolfram Mathematica 9.0 represents a proper comparative of entropy in continuous terms. Frequency distributions of three, four, five and six polygons are the four lowest values which is an indicative of low entropy since the KST value is directly proportional to the distance between continual and normal distribution (less distance less entropy). Whereas there is an incremental closeness in frequency distributions with a comparative normal distribution except for shapes $\Gamma$ with four sides. Also, as an initial experiment one BIO sample (PSD); [40] and one random sample are included showing that BIO sample has an important contrast with random sample in terms of distance. The BIO sample reach a value of -38.54 while the random value is -1.23 . 


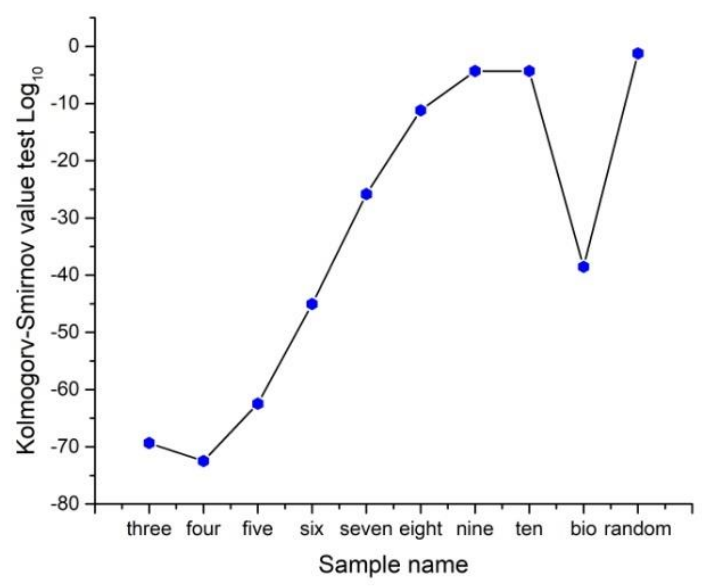

Figure 5. Continuous distributions of frequencies using heterogeneity data. In order to start with a continuous approach to detect levels of entropy we use KolmogorovSmirnov test (KST) as a parameter to detect distribution differences between normal distributions and the remaining ones. According to the Log base 10 values of Kolmogorov Smirnov test applied, three, four, five and six partition number frequency distributions have the lowest values of entropy in continuous terms. The KST value is directly proportional to the distance between distributions. Lowest values of the KST are indicative of lowest entropy. Random sample have the closest value to normal distribution.

\subsection{Binarization of frequencies as discrete variables.}

The Shannon entropy is a parameter indicating a degree of information approaching a resolution of uncertainty. Our model satisfies the fact that we may work with frequencies of numerical variables, which are sub-areas of polygonal shapes. Shannon elucidates the convenience of the use of a logarithmic function in the definition of entropy, mainly because "it is more suitable" mathematically, due to the fact that many operations in terms of the logarithm are simpler than in terms of the statistical behavior (the number of possibilities or frequency). In fact, one of our main source ideas is the finding of a practical procedure to retrieve polygonally shape patterns (PSP) given an entropy value. The average surprise of a variable $X$ which has a distribution $p(X)$ is called the entropy of $p(X)$, and is represented as $H(X)$. For convenience, we often speak of the entropy of the variable $X$, even though, strictly speaking, entropy refers to the distribution $p(X)$ of $X$ [56]. Thus, the entropy of our $x_{i}$ partition and locality values can be derived from the general formula:

$$
H(X) \approx \frac{1}{N_{i}} \sum_{i=1}^{N_{i}} \frac{1}{p\left(x_{i}\right)}
$$

The choice of a logarithmic base regards for a proper election of a unit for measuring information. In consonance with this last idea we consider frequency values of heterogeneity in a range of bin variables. The entropy values using different bin categorizations and their associated entropy (bin 0.1 , bin 0.2 , bin 0.25 , bin 0.33 and bin 0.5) shown that the binarization (bin 0.5) of the model influences the results giving a clear difference with the remaining ones (Figure 6). Our finding here regards for the lowest values in terms of entropy. On the other hand, it is important to inspection how close is the bin 0.25 function of entropy with that of standard deviation heterogeneity of figure 4 . Nevertheless, we consider low entropy measure 
(binarization) as a solid argument to be applied not just on individual polygons but to explore whether or not there are entropy differences between more complex biological and non-biological organizations in terms of entropy.

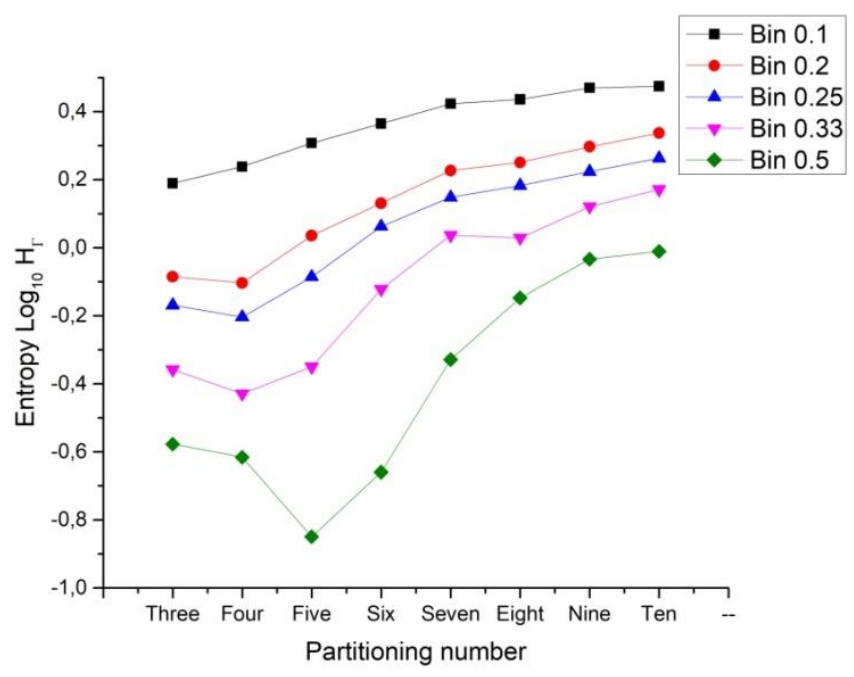

Figure 6. Binarization of entropy $H_{\Gamma}$. Partitioning number and their associated entropy can be derived from different bin statistical discrete categorization. Graphic shows five bin categorizations and their associated entropy (bin 0.1, bin 0.2, bin 0.25, bin 0.33 and bin 0.5 ). Bin 0.5 has the lowest values of entropy for every partitioning number. The entropy of shapes gamma $H_{\Gamma}$ using the logarithm to the base 10 indicates that partition number five has the lowest values of entropy in a binarized system (green line). Extreme low values are founded for 3, 4 and 6 organizations in a binarized system. Bin 0.1 statistical categorization has a linear incremental behavior in contrast with the remaining categorizations. The highest values of entropy are incremental from 5 to 10 disc organizations in a binarized system.

The nomenclature of discrete variables could be defined as a binary result if the outcomes for heterogeneity levels (equation 3) are tagged as follows:

$$
\begin{gathered}
0 \leq x_{i}<0.5 \Rightarrow X_{i}=x_{i l} \\
0.5 \leq x_{i} \leq 1 \Rightarrow X_{i}=x_{i h}
\end{gathered}
$$


With these definitions, one bit of information would be obtained when one of two equally likely alternatives is specified. Using this as reference the Shannon entropy equation will define the entropy in terms of the frequency of two possibilities $x_{i l}$ and $x_{i h}$, where the first subscript $l$ mean low heterogeneity values between 0 and $0, .5$ and the second subscript $h$ implies high heterogeneity values between 0.5 and 1 . Therefore, we can use a well known entropy equation typically used to determine the uncertainty of binary outcomes:

$$
H_{\Gamma}(X)=p\left(x_{i l}\right) \log _{2}\left(\frac{1}{p\left(x_{i l}\right)}\right)+p\left(x_{i h}\right) \log _{2}
$$

In order to link entropy and geometrical information, in this section our evidence suggests that five-folding organization depicts a sort of spatial organization with low values of information without reach the zero value, besides three, four and six folding organizations which are also frequent in nature.

\subsection{Statistical distributions of internal partition in shapes $\Gamma$ and localities in Bio, Non-Bio and RA samples.}

According to partition number the statistical frequency distribution of heterogeneity of shapes $\Gamma$ is showed in figure 7. The bar area determines levels of heterogeneity conforming the binary categorization $x_{i l}$ and $x_{i h}$ described in equation 6 . The green area shows levels of heterogeneity where $0.5 \leq x_{i}<1 \Rightarrow X_{i}=x_{i l}$ for standard deviation indexes derived from equation 3, which implies low levels of heterogeneity. It is clear that five-folding organizations have the lowest values for heterogeneity (green area) indexes indicating also highest level of homogeneity (grey area; $\left.0 \leq x_{i}<0.5 \Rightarrow X_{i}=x_{i h}\right)$. In fact, this figure reflects the performance of spatial organizations that have been shown shapes $\Gamma$ in figure 4 and 5 . Regarding to this last point, it is important to focus on the fact that now we have these two necessary outcomes for heterogeneity which were used to define the entropy of internal partitioning in shapes $\Gamma$ using equation (7):

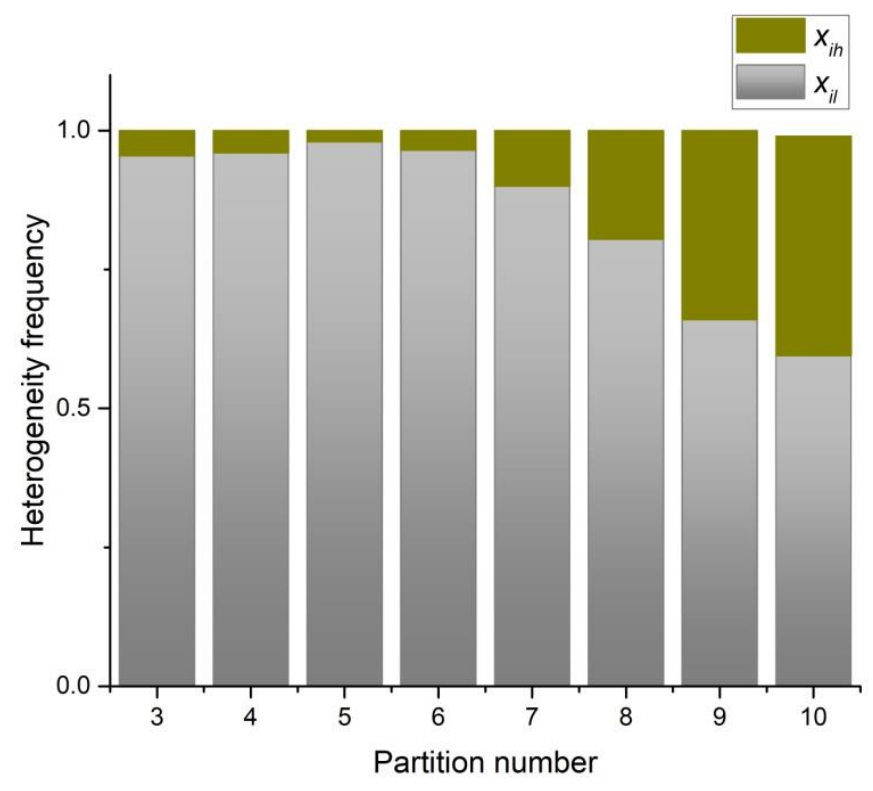


Figure 7. Levels of heterogeneity according to the binary categorization $x_{i l}$ and $x_{i h}$. Grey zones are values associated to $x_{i l}$ and the green ones are associated with $x_{i h}$. The highest level of homogeneity is for partition number five, even though, three, four and six have similar levels. The highest level of heterogeneity is for partitioning number ten.

In order to link entropy and geometrical information, our evidence suggests that fivefolding organization depicts a sort of spatial organization with low values of information, besides three, four and six folding organizations which are also frequent in nature with low values of entropy in all bin categorizations (Figure 6). Consequently, we have establish a measure of spatial organization able to determine the geometrical entropy of a particular set of forms. In that sense, it is not surprising that figure 7 is intrinsically related with the binarization of entropy in figure 6 . Therefore, the low spatial variability average for five-fold morphology reflects a high degree of homogeneity in terms of their internal spatial distribution. As we have said before, the fact that five-fold partitions reflect the most equal distribution of internal space in contrast with other partitions it is a non trivial result since this is not a function derived from the polygonal number of sides. On the other hand, the binarization of frequencies represents a simple method based on practical and heuristic motivations to build collective polygonal arrangements simulating biological arrangements since we only requires a binary distribution based on heterogeneity of individual polygons. Biological organizations of cell aggregates that were derived directly from natural images or even simulations have a constant high proportion of homogeneity in terms of spatial distribution. A clear high degree of homogeneity is found in all samples derived from biological approximations (Figure 8).

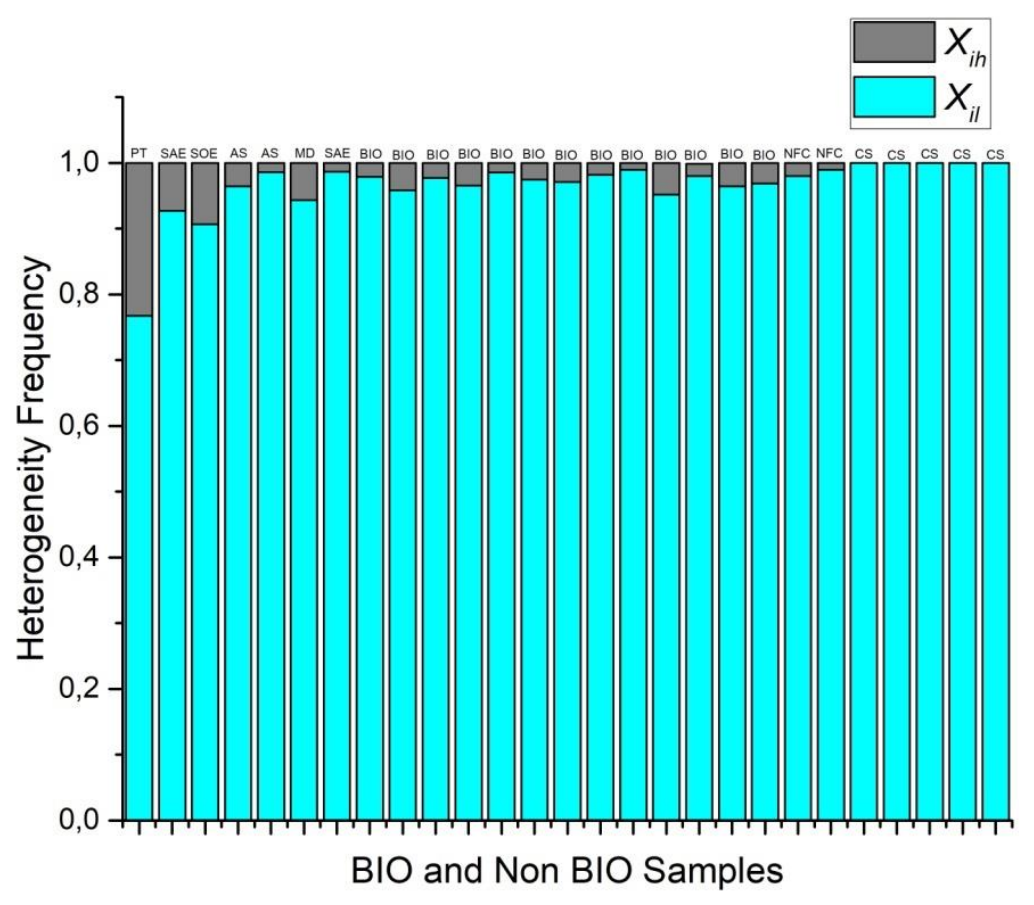


Figure 8. Twenty-seven samples of biological and biological simulations of organizations of cells aggregates have a constant high proportion of homogeneity in terms of spatial distribution of inner areas (For data related with samples see Supplementary digital content 1). In fact, the last five samples are biological simulations assuming Lewis's Law with a $100 \%$ degree of homogeneity. The first column represents a Poisson-Voronoi Tesselation used as control.

In terms of the entropy values using binarization there is a limited range where all biological samples fall into. We call it the BIO zone (Figure 9, between 0.08 and 0.27). The BIO zone is composed of 16 samples whose descriptions are showed in table 1 (Supplementary digital content). There are an important number of simulations extracted from the web that are excluded from the BIO zone. All of those collected simulations were based on quantified distances from SDCP conserved in proliferating metazoans tissues with a polygonal frequency of $49 \%$ of 6 -sided polygons, $29 \%$ of 5 -sided polygons, and $20 \%$ 7-sided polygons, which is traditionally used as reference in epithelial studies. Control simulation (CS), simulation at equilibrium (SAE), atrophy simulation (AS) and simulation out of equilibrium (SOE) were computational simulations of cells aggregates assuming variations as metric distances from Lewis's Law values or another kind of biological or physical properties. In spite of the idea of being biological simulations, our results shown that these images are detected as non-biological samples (Figure 10) since most of them are non-heterogeneous at all, except for AS and MD. The Shannon entropy associated to biological simulations (CS) reaches levels of zero entropy, which imply null information which is not the case for both partition discs associated with biological morphologies and for that samples constricted to the BIO zone. Certainly, PoissonVoronoi tessellation (PT) was used as control since we consider that its arrangement would be far away from the order zone. Hence, the geometry between biological and non-biological arrangements of internal cells is underlying an important difference whose consequences and effects would define particular behavior in biological organizations in contrast with non-biological packages.

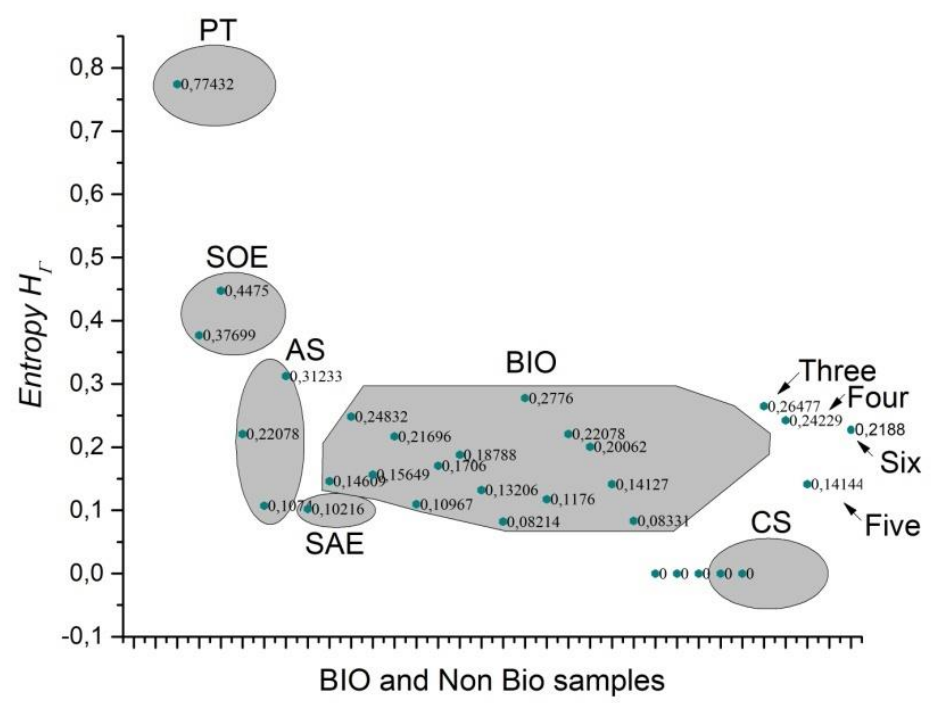

Figure 9. The entropy of cell aggregates groups: biological collected images directly from the web (BIO; descriptions in table 1) and processed images extracted from the web which we named control simulation (CS), simulation at equilibrium following four interations of Lloyd's algorithm (SAE), atrophy simulation (AS), simulation out 
of equilibrium (SOE), muscular distrophy (MD) and Poisson-Voronoi tessellation (PT). The most abundant area includes Bio data and it also can include AS.

The Shannon entropy associated with RA frequency distribution (Figura 10) is an expected result whose entropy values are around 1 (Figure 11). This result is a clear support to argue the value of approaching the geometry of biological organizations with the binarization method. Figure 10 and its associated entropy at figure 11 indicates that binarizations works well as an indicator of informational limits in complex meshes of polygonal arrangements since those two figures perform a control experiment to visualize contrast in terms of distributions and their associated Shannon entropy.

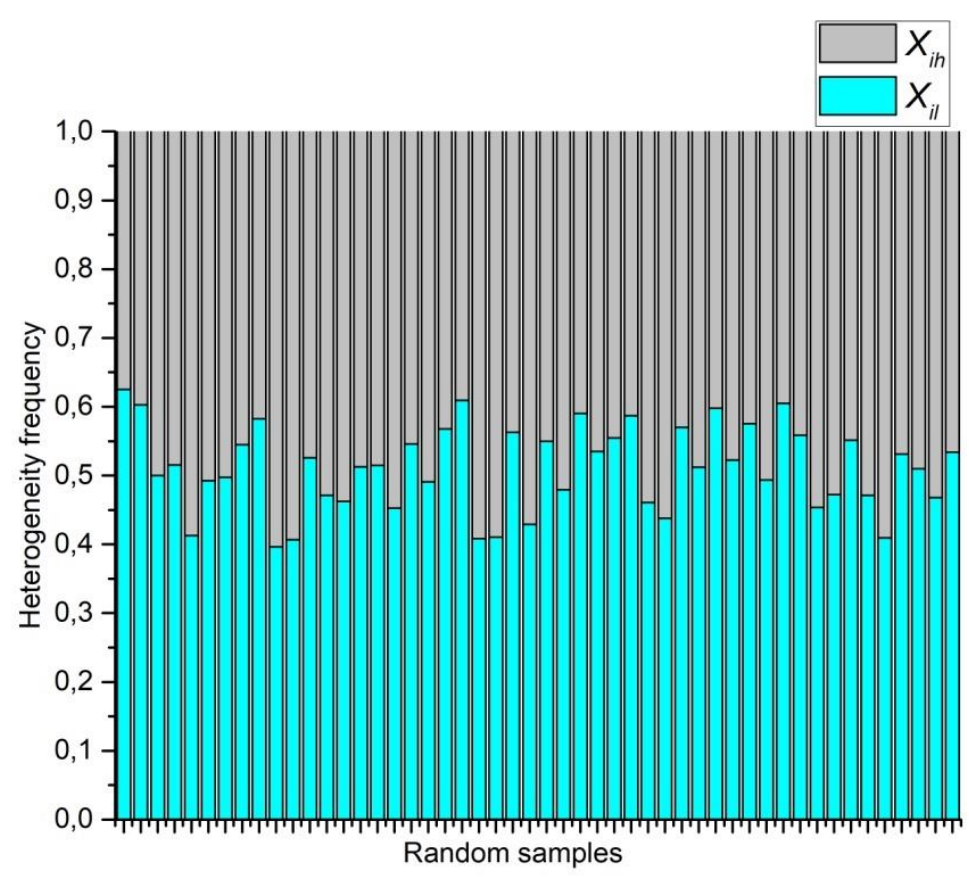

Figure 10. Random arrangements of cells and their heterogeneity frequency. Data shows that cellular aggregates have an average of an almost half proportion of heterogeneity (blue) of spatial distribution on internal areas in polygons, and a half of spatial homogeneity (grey). 


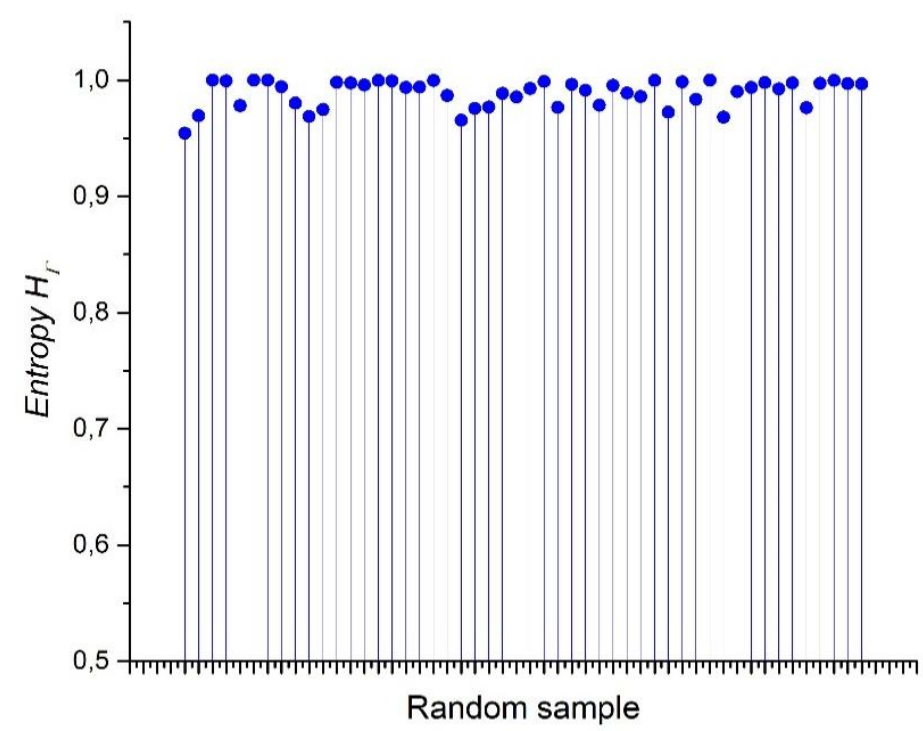

Figure 11. The entropy of random samples. The entropy values are almost constantly in line with maximum entropy.

\section{Discussion}

We may see that three, four, five-fold and six morphologies behave as almost egalitarians in terms of internal spatial distribution with a possible parametrical impact on the collective behavior of geometry in biological organizations. Partition number and their associated entropy can be derived from different bin statistical discrete categorization. In figure 6, graphic shows five bin categorizations and their associated entropy (bin 0.1 , bin 0.2 , bin 0.25 , bin 0.33 and bin 0.5 ). Bin 0.5 has the lowest values of entropy for every partitioning number and five-fold partitions is at the lowest point of binary categorizations. Therefore, the entropy of shapes gamma $\boldsymbol{H}_{\boldsymbol{\Gamma}}$ indicates that partitioning number five has the lowest values of entropy in a binarized system. Similar values are founded for 3,4 and 6 organizations. On the other side, bin 0.1 statistical categorization has a linear incremental behavior in contrast with the remaining categorizations. The final decision of using a binarized system to determine entropy was implemented in meshes of different polygonal organizations. The highest level of homogeneity for partitioning number five into the binarized system represents the establishment of a biological form of reference, which we establish to determine the geometrical entropy of a particular biological form. In addition, we stated [30] that the significance of our findings was based on the statistical constancy of geometrical constraints which eventually would motivate a more generic approach using geometry as a source of information. According to our binary partition of frequency distribution (where above of 0 and below of 0.5 of heterogeneity represents $\boldsymbol{X}_{\boldsymbol{i}}$ and above of 0.5 of heterogeneity but below of 1 represents $\boldsymbol{X}_{i h}$ ) it is clear that five-folding arrangements distributes internal space in a very equal statistical way. In terms of entropy derived from equation 6 five-folding partition reach the lowest point with a Log value of -0.06 (Figure 6). This finding is a nontrivial fact, since we should not expect statistical differences between partitions if the source of variation is a random source and equal variability is included in all analyzed samples. Therefore, we should expect no difference at all since it is a random experiment just varying partitioning. Using this argument as reference a gap of entropy values emerges clustering arrangements from biological samples (around 0.08 and 0.25 of entropy). To do this, three types of mosaics were analyzed: Random arrangements (RA), natural images extracted from the web (BIO) and processed images also extracted from the web, which we named control simulation (CS), simulation at equilibrium (SAE Ll'A), atrophy simulation (AS), simulation out of 
equilibrium (SOE) and Poisson-Voronoi tessellation (PT). Spatial heterogeneity in mosaics of polygons was derived using equation (3) for each polygon and entropy using equation (6). Random arrangements of cells and their heterogeneity frequency shows that random polygonal aggregates representing cell aggregates have an average of an almost half proportion of heterogeneity of spatial distribution on internal areas in polygons with a nearby equal half of spatial homogeneity. Biological and biological simulations (which we has included as part of that non-biological samples) of organizations of cells aggregates have a constant high proportion of homogeneity in terms of spatial distribution of inner areas. Some other approaches have found similar results, such as that analyzing avian photoreceptor patterns representing a disordered hyperuniform solution to a multiscale packing problem [55]. In fact, the last five samples areas in biological simulations assuming Lewis's Law have a $100 \%$ degree of homogeneity. Then, a high degree of homogeneity in a computational simulation following some algorithmic instructions could derive in a beautiful representation following the SDCP of a real biological sample but a considerable lacking of substantive geometric information. Thus, levels of intrinsic disorder emerging from the actual biological forms are necessary to have a proper simulation. On the other hand, BIO group is around 0.08 and 0.27 which is a range for entropy values including three, four, five and six folding partitions which are very common in nature. Also, in figure 8 and 9 the first value represents a Poisson-Voronoi Tesselation (PT) which was used as a control since this mesh is derived from a nonordered organization of points. Even this sort of organization is not biological at all it seems non close to random organizations. The most abundant area in bars of figure 9 appears on BIO data, which also include AS. We conclude that the atrophy of some simulations increase its heterogeneity degree which finally derives in a biological outcome. The inclusion of them into BIO group is not an unexpected result since computational simulations representing algorithmic instructions are perturbed in a way that could easily increase their entropy. It does not happen with control simulations. Those algorithmic constructions are following mathematical prescriptions whose level of homogeneity leaves out the fundamental nature of BIO group (i.e. a lightly bias disruption of order). In fact, five control simulation group whose main feature has been the closeness with SDCP has a value of zero of entropy (Figure $8 \& 9$ ). On the other hand, MD seems to be a close object to BIO realm. However, it is not inside the limits. We consider that it is an important find since our parametric measure of geometric information can give us some clues about pathological routes in a very simplistic way, that important finding is in agreement with [37]. At the level of ecological scales, we include just two samples that were very representative. Namibia fairy circles are one of the most interesting results since we confirmed some previous hypothesis about the potential of free scale approaches to understand biological organizations [50].

\section{Conclusions}

Our results reflect that there is a potential informational limit for biological organizations in terms of binarization and Shannon entropy. According to this conclusion, biological organizations are complex systems which should be constrained into a narrow window of variability depending on levels of informational entropy. However, the fact is that we can see a myriad of morphological variations in nature. We conclude that the statistical properties of biological architectures can be manifested into an overwhelming amount of morphologies since all of them are singular possibilities in a realm of pure organization with particular geometrical attributes. In that sense, shape is a constant dynamical composition of arrangements and an opening infinite possibility of configurations with biological attributes as a consequence of its essential organization which depends on their own informational limits. According to our results, we consider that homogeneity with low levels of heterogeneity in biological systems is a fundamental factor. Network theory calls it sparsity. In the context of complex adaptive systems heterogeneity could be associated with a source of variation 
which is notably different from the genetic approach. With this in mind, we consider that the value and limits of informational entropy for geometrical systems that we are approaching has a width domain of impact.

Author Contributions: Conceptualization, Juan Lopez-Sauceda and Philipp von Bülow; methodology, Juan Lopez-Sauceda, Philipp von Bülow, Jose Gerardo Carrillo Gonzalez, Carlos Ortega Laurel, Gerardo Laguna Sanchez, Jacobo Sandoval Gutierrez, Francisco PerezMartinez and Kalina Miranda-Perkins; software, Juan Lopez-Sauceda, Philipp von Bülow, Jose Gerardo Carrillo Gonzalez, Gerardo Laguna Sanchez; validation, Jose Gerardo Carrillo Gonzalez, Carlos Ortega Laurel, Gerardo Laguna Sanchez, Jacobo Sandoval Gutierrez, Francisco Perez-Martinez and Kalina Miranda-Perkins; formal analysis, Philipp von Bülow, Juan Lopez-Sauceda and Jose Gerardo Carrillo Gonzalez; investigation, Juan Lopez-Sauceda, Jose Gerardo Carrillo Gonzalez, Carlos Ortega Laurel, Gerardo Laguna Sanchez, Jacobo Sandoval Gutierrez, Francisco Perez-Martinez; resources, Juan Lopez-Sauceda, Philipp von Bülow, Jose Gerardo Carrillo Gonzalez, Carlos Ortega Laurel, Gerardo Laguna Sanchez, Jacobo Sandoval Gutierrez, Francisco Perez-Martinez and Kalina Miranda-Perkins; data curation, Juan Lopez-Sauceda, Philipp von Bülow, and Jose Gerardo Carrillo Gonzalez; writing - Juan Lopez-Sauceda, Philipp von Bülow, and Jose Gerardo Carrillo Gonzalez; writing-review and editing, Juan Lopez-Sauceda, Philipp von Bülow, Carlos Ortega Laurel, Gerardo Laguna Sanchez, Jacobo Sandoval Gutierrez, Francisco Perez-Martinez and Kalina Miranda-Perkins; visualization, Juan Lopez-Sauceda, Carlos Ortega Laurel, Jacobo Sandoval Gutierrez, Francisco Perez-Martinez and Kalina Miranda-Perkins; supervision, Juan LopezSauceda; project administration, Juan Lopez-Sauceda; funding acquisition, Juan LopezSauceda, Philipp von Bülow, Jose Gerardo Carrillo Gonzalez, Carlos Ortega Laurel, Gerardo Laguna Sanchez, Jacobo Sandoval Gutierrez, Francisco Perez-Martinez and Kalina MirandaPerkins.

Funding: "This research received no external funding"

Conflicts of Interest: “The authors declare no conflict of interest.”

\section{References}

1. M. A. G. Valerio, “Agenciamientos materiales y formales. Variaciones sobre morfología,” Azafea Rev. Filos., vol. 19, no. 1, pp. 63-89, 2017. [Online]. Available: http://revistas.usal.es/index.php/0213-3563/article/view/17407

2. C. Alexander, "A vision of a living world: The nature of order, book 3: An essay of the art of building and the nature of the universe.", $3^{\text {rd }}$ ed., United Kingdom: Berkeley: Routledge, 2005, pp. 75-102.

3. A. Nematbakhsh et al., "Multi-scale computational study of the mechanical regulation of cell mitotic rounding in epithelia," Plos Computational Biology, vol. 13, no. 5, May 2017, Art no. e1005533, doi: 10.1371/journal.pcbi.1005533.

4. L. Atia et al., "Geometric constraints during epithelial jamming," Nature Physics, vol. 14, no. 6, pp. 613-620, Jun 2018, doi: 10.1038/s41567-018-0089-9.

5. G. van Anders, D. Klotsa, N. K. Ahmed, M. Engel, and S. C. Glotzer, "Understanding shape entropy through local dense packing," Proceedings of the National Academy of Sciences of the United States of America, vol. 111, no. 45, pp. E4812-E4821, Nov 2014, doi: 10.1073/pnas.1418159111.

6. $\quad$ A. Tsuboi et al., "Competition for Space Is Controlled by Apoptosis-Induced Change of Local Epithelial Topology," Current Biology, vol. 28, no. 13, pp. 2115-+, Jul 2018, doi: 10.1016/j.cub.2018.05.029. 
7. A. Gough et al., "Biologically Relevant Heterogeneity: Metrics and Practical Insights," Slas Discovery, vol. 22, no. 3, pp. 213237, Mar 2017, doi: 10.1177/2472555216682725.

8. C. Brandl et al., "In-Depth Characterisation of Retinal Pigment Epithelium (RPE) Cells Derived from Human Induced Pluripotent Stem Cells (hiPSC)," Neuromolecular Medicine, vol. 16, no. 3, pp. 551-564, Sep 2014, doi: 10.1007/s12017-014-83088.

9. E. Boghaert, J. P. Gleghorn, K. Lee, N. Gjorevski, D. C. Radisky, and C. M. Nelson, "Host epithelial geometry regulates breast cancer cell invasiveness," Proceedings of the National Academy of Sciences of the United States of America, vol. 109, no. 48, pp. 19632-19637, Nov 2012, doi: 10.1073/pnas.1118872109.

10. M. R. V Sahyun, “Aesthetics and Entropy III. Aesthetic Measures," 2018. [Online]. Available: https://www.preprints.org/manuscript/201801.0098/v1.Doi:10.20944/preprints201801.0098.v1. Accessed on: June 8, 2019.

11. A. M. Lopes and J. A. T. Machado, "Complexity Analysis of Escher's Art," Entropy, vol. 21, no. 6, Jun 2019, doi: $10.3390 / \mathrm{e} 21060553$.

12. B. Goodwin, How the leopard changed its spots: The evolution of complexity, $18^{\text {th }}$ ed., vol. 24. Princeton, New Jersey, USA: Princeton University Press, 2001, pp. 189-202.

13. C. P. Pipinikas, A. M. Berner, T. Sposito, and C. Thirlwell, "The evolving (epi)genetic landscape of pancreatic neuroendocrine tumours," Endocrine-Related Cancer, vol. 26, no. 9, pp. R519-R544, Aug 2019, doi: 10.1530/erc-19-0175.

14. S. Douady and Y. Couder, "PHYLLOTAXIS AS A PHYSICAL SELF-ORGANIZED GROWTH-PROCESS," Physical Review Letters, vol. 68, no. 13, pp. 2098-2101, Mar 1992, doi: 10.1103/PhysRevLett.68.2098.

15. G. Oster and P. Alberch, "EVOLUTION AND BIFURCATION OF DEVELOPMENTAL PROGRAMS," Evolution, vol. 36, no. 3, pp. 444-459, 1982, doi: 10.2307/2408093.

16. D. M. Busiello, S. Suweis, J. Hidalgo, and A. Maritan, "Explorability and the origin of network sparsity in living systems," Scientific Reports, vol. 7, Sep 2017, Art no. 12323, doi: 10.1038/s41598-017-12521-1.

17. J. Demongeot, M. Jelassi, H. Hazgui, S. Ben Miled, N. B. Ben Saoud, and C. Taramasco, "Biological Networks Entropies: Examples in Neural Memory Networks, Genetic Regulation Networks and Social Epidemic Networks," Entropy, vol. 20, no. 1, Jan 2018, Art no. 36, doi: 10.3390/e20010036.

18. G. Bianconi, "The entropy of randomized network ensembles," Epl, vol. 81, no. 2, 2008, Art no. 28005, doi: 10.1209/0295$5075 / 81 / 28005$.

19. L. Demetrius and T. Manke, "Robustness and network evolution - an entropic principle," Physica a-Statistical Mechanics and Its Applications, vol. 346, no. 3-4, pp. 682-696, Feb 2005, doi: 10.1016/j.physa.2004.07.011. 
landscape entropy," Landscape Ecology, vol. 30, no. 1, pp. 7-10, Jan 2015, doi: 10.1007/s10980-014-0108-x.

21. I. Vranken, J. Baudry, M. Aubinet, M. Visser, and J. Bogaert, "A review on the use of entropy in landscape ecology: heterogeneity, unpredictability, scale dependence and their links with thermodynamics," Landscape Ecology, vol. 30, no. 1, pp. 51-65, Jan 2015, doi: 10.1007/s10980-014-0105-0.

22. L. Parrott, "Measuring ecological complexity," Ecological Indicators, vol. 10, no. 6, pp. 1069-1076, Nov 2010, doi: 10.1016/j.ecolind.2010.03.014.

23. R. Proulx and L. Parrott, "Measures of structural complexity in digital images for monitoring the ecological signature of an old-growth forest ecosystem," Ecological Indicators, vol. 8, no. 3, pp. 270-284, May 2008, doi: 10.1016/j.ecolind.2007.02.005.

24. N. J. Frost, M. T. Burrows, M. P. Johnson, M. E. Hanley, and S. J. Hawkins, "Measuring surface complexity in ecological studies," Limnology and Oceanography-Methods, vol. 3, pp. 203-210, Apr 2005, doi: 10.4319/lom.2005.3.203.

25. P. C. W. Davies, E. Rieper, and J. A. Tuszynski, "Self-organization and entropy reduction in a living cell," Biosystems, vol. 111, no. 1, pp. 1-10, Jan 2013, doi: 10.1016/j.biosystems.2012.10.005.

26. A. B. C. Buskermolen et al., "Entropic Forces Drive Cellular Contact Guidance," Biophysical Journal, vol. 116, no. 10, pp. 19942008, May 2019, doi: 10.1016/j.bpj.2019.04.003.

27. P. Cabral, G. Augusto, M. Tewolde, and Y. Araya, "Entropy in Urban Systems," Entropy, vol. 15, no. 12, pp. 5223-5236, Dec 2013, doi: 10.3390/e15125223.

28. C. Gershenson and N. Fernandez, "Complexity and information: Measuring emergence, self-organization, and homeostasis at multiple scales," Complexity, vol. 18, no. 2, pp. 29-44, Nov-Dec 2012, doi: 10.1002/cplx.21424.

29. H. A. Martinez-Berumen, G. C. Lopez-Torres, and L. Romo-Rojas, "Developing a Method to Evaluate Entropy in Organizational Systems," 2014 Conference on Systems Engineering Research, vol. 28, pp. 389-397, 2014, doi: 10.1016/j.procs.2014.03.048.

30. J. Lopez-Sauceda, J. Lopez-Ortega, G. A. Laguna Sanchez, J. S. Gutierrez, A. P. R. Meza, and J. L. Aragon, "Spatial Organization of Five-Fold Morphology as a Source of Geometrical Constraint in Biology," Entropy, vol. 20, no. 9, Sep 2018, Art no. 705, doi: 10.3390/e20090705.

31. P. Gomez-Galvez et al., "Scutoids are a geometrical solution to three-dimensional packing of epithelia," Nature Communications, vol. 9, Jul 2018, Art no. 2960, doi: 10.1038/s41467-018-05376-1.

32. M. A. Klatt et al., "Universal hidden order in amorphous cellular geometries," Nature Communications, vol. 10, Feb 2019, Art no. 811, doi: 10.1038/s41467-019-08360-5.

33. K. A. Rejniak et al., "Linking Changes in Epithelial Morphogenesis to Cancer Mutations Using Computational Modeling," Plos Computational Biology, vol. 6, no. 8, Aug 2010, Art no. e1000900, doi: 10.1371/journal.pcbi.1000900. 
34. D. Sanchez-Gutierrez, M. Tozluoglu, J. D. Barry, A. Pascual, Y. Mao, and L. M. Escudero, "Fundamental physical cellular constraints drive self-organization of tissues," Embo Journal, vol. 35, no. 1, pp. 77-88, Jan 2016, doi: 10.15252/embj.201592374.

35. S. A. Sandersius, M. Chuai, C. J. Weijer, and T. J. Newman, "Correlating Cell Behavior with Tissue Topology in Embryonic Epithelia," Plos One, vol. 6, no. 4, Apr 2011, Art no. e18081, doi: 10.1371/journal.pone.0018081.

36. G. A. Stooke-Vaughan and O. Campas, "Physical control of tissue morphogenesis across scales," Current Opinion in Genetics E Development, vol. 51, pp. 111-119, Aug 2018, doi: 10.1016/j.gde.2018.09.002.

37. L. Yan and D. P. Bi, "Multicellular Rosettes Drive Fluid-solid Transition in Epithelial Tissues," Physical Review X, vol. 9, no. 1, Feb 2019, Art no. 011029, doi: 10.1103/PhysRevX.9.011029.

38. E. Bormashenko et al., "Characterization of Self-Assembled 2D Patterns with Voronoi Entropy," Entropy, vol. 20, no. 12, Dec 2018, doi: 10.3390/e20120956.

39. C. J. Wang and H. R. Zhao, "Spatial Heterogeneity Analysis: Introducing a New Form of Spatial Entropy," Entropy, vol. 20, no. 6, Jun 2018, Art no. 398, doi: 10.3390/e20060398.

40.

W. T. Gibson and M. C. Gibson, "CELL TOPOLOGY, GEOMETRY, AND MORPHOGENESIS IN PROLIFERATING EPITHELIA," Tissue Remodeling and Epithelial Morphogenesis, vol. 89, pp. 87-114, 2009, doi: 10.1016/s0070-2153(09)89004-2.

41. C. E. Shannon, "A MATHEMATICAL THEORY OF COMMUNICATION," Bell System Technical Journal, vol. 27, no. 3, pp. 379-423, 1948, doi: 10.1002/j.1538-7305.1948.tb01338.x.

42. G. Nicolis, "Self-organization in nonequilibrium systems," Dissipative Struct. to Order through Fluctuations, 1 st ed., Hoboken, New Jersey, USA: Wiley, 1977, pp. 10-135.

43. Y. L. Klimontovich, "Turbulent Motion. The Structure of Chaos," in Turbulent Motion and the Structure of Chaos, $1^{\text {st }}$ ed., Berlin, Germany: Springer, 1991, pp. 329-371.

44. M. I. Drag, "Epithelium: The lightweight, customizable epithelial tissue simulator," Ph.D. thesis, The Ohio State University, 2015. [Online]. Available: https://etd.ohiolink.edu/!etd.send file?accession=osu1430011382\&disposition=inline. Accessed on: March 6, 2019

45. D. Sanchez-Gutierrez, A. Saez, A. Pascual, and L. M. Escudero, "Topological Progression in Proliferating Epithelia Is Driven by a Unique Variation in Polygon Distribution," Plos One, vol. 8, no. 11, Nov 2013, Art no. e79227, doi: 10.1371/journal.pone.0079227.

46. A. Saez et al., "Quantifiable diagnosis of muscular dystrophies and neurogenic atrophies through network analysis," Bmc Medicine, vol. 11, Mar 2013, Art no. 77, doi: 10.1186/1741-7015-11-77.

47. L. M. Escudero, L. D. Costa, A. Kicheva, J. Briscoe, M. Freeman, and M. M. Babu, "Epithelial organisation revealed by a network of cellular contacts," Nature Communications, vol. 2, Nov 2011, Art no. 526, doi: 10.1038/ncomms1536. 
48. F. Pilot and T. Lecuit, "Compartmentalized morphogenesis in epithelia: From cell to tissue shape," Developmental Dynamics, vol. 232, no. 3, pp. 685-694, Mar 2005, doi: 10.1002/dvdy.20334.

49. J. Lopez-Sauceda and M. D. Rueda-Contreras, "A Method to Categorize 2-Dimensional Patterns Using Statistics of Spatial Organization," Evolutionary Bioinformatics, vol. 13, Mar 2017, Art no. 1176934317697978, doi: 10.1177/1176934317697978.

50. H. Z. Zhang and R. Sinclair, "Namibian fairy circles and epithelial cells share emergent geometric order," Ecological Complexity, vol. 22, pp. 32-35, Jun 2015, doi: 10.1016/j.ecocom.2015.02.001.

51. S. Getzin, K. Wiegand, T. Wiegand, H. Yizhaq, J. von Hardenberg, and E. Meron, "Adopting a spatially explicit perspective to study the mysterious fairy circles of Namibia," Ecography, vol. 38, no. 1, pp. 1-11, Jan 2015, doi: 10.1111/ecog.00911.

52. M. C. Gibson, A. B. Patel, R. Nagpal, and N. Perrimon, "The emergence of geometric order in proliferating metazoan epithelia," Nature, vol. 442, no. 7106, pp. 1038-1041, Aug 2006, doi: 10.1038/nature05014.

53. R. Nagpal, A. Patel, and M. C. Gibson, "Epithelial topology," Bioessays, vol. 30, no. 3, pp. 260-266, Mar 2008, doi: 10.1002/bies.20722.

54. A. B. Patel, W. T. Gibson, M. C. Gibson, and R. Nagpal, "Modeling and Inferring Cleavage Patterns in Proliferating Epithelia," Plos Computational Biology, vol. 5, no. 6, Jun 2009, Art no. e1000412, doi: 10.1371/journal.pcbi.1000412.

55. Y. Jiao, T. Lau, H. Hatzikirou, M. Meyer-Hermann, J. C. Corbo, and S. Torquato, "Avian photoreceptor patterns represent a disordered hyperuniform solution to a multiscale packing problem," Physical Review E, vol. 89, no. 2, Feb 2014, Art no. 022721, doi: 10.1103/PhysRevE.89.022721.

56. J. V Stone, Information theory: a tutorial introduction. Sebtel Press, 2015. 